\title{
Thin films of AlCrFeCoNiCu high-entropy alloy by pulsed laser deposition
}

M. D. Cropper

Department of Physics, Loughborough University,

Loughborough

LE11 3TU, UK.

m.d.cropper@lboro.ac.uk

Pulsed laser deposition has been used to prepare thin films of the high entropy alloy AlCrFeCoNiCu. The $35 \mathrm{~nm}$ films were deposited in ultra-high vacuum onto glass at room temperature and above and analysed using X-ray diffraction and X-ray photoelectron spectroscopy. Films deposited at room temperature exhibit a mix of FCC and BCC reflections, the FCC crystallites having size similar to the film thickness, but the BCC crystallites are larger. The intensity of the reflections from both crystal structures reduce with increasing deposition temperature, the fall in BCC commencing at lower temperature than the FCC associated with a reduction of the content of $\mathrm{Al}$ and $\mathrm{Cu}$. X-ray photoelectron spectroscopy shows that the films deposited at room temperature are closer to stoichiometry than those at higher temperatures. An important feature of the X-ray photoelectron spectroscopy depth profiles is surface segregation, the outer $3 \mathrm{~nm}$ of the high entropy alloy films has higher concentration of $\mathrm{Al}$ and, to a lesser extent, $\mathrm{Cr}$.

\section{Highlights}

- $\quad$ The films exhibit mixed FCC and BCC structure.

- With increasing deposition temperature, FCC and BCC reflections reduce associated with loss of $\mathrm{Al}$ and $\mathrm{Cu}$.

- Surface segregation enriches the surface of the films in Al and to a lesser extent Cr.

Keywords: High entropy alloy; AICrFeCoNiCu; PLD; XRD; XPS. 


\section{Introduction}

High entropy alloys (HEA) are alloys with at least five components that are approximately equi-molar [1-3]. They tend to form solid solutions with FCC and/or BCC lattices, stabilised by the entropy of mixing. There is a burgeoning body of work discussing the properties of these materials inspired primarily by their impressive mechanical properties.

In addition to the large literature relating to the properties of these alloys in the bulk, there are an increasing number of reports about the deposition of thin films of high entropy alloys using physical vapour deposition, specifically DC and RF sputtering. These are focussed on mechanical properties [4-6], structure and morphology [7], electrical properties [8], and magnetic properties [9]. There has been a particular focus on thin films of the HEA CrFeCoNiCu, with [10-15] or without a sixth component $[16,17]$.

A study of sputter deposition of AlCrFeCoNiCu films from mosaic targets [15] reported that the structure could change between solid solutions of FCC, BCC or disordered depending on quite small changes in composition, BCC stabilised by Al and $\mathrm{Cr}, \mathrm{FCC}$ by $\mathrm{Cu}$, Co and $\mathrm{Ni}$. A related study showed a mixed FCC, BCC structure that was stable on heating to $310^{\circ} \mathrm{C}$ on an Si substrate and an FCC that was stable to $510^{\circ} \mathrm{C}$.

More recent work employing pressed powder targets has examined the influence of the sixth element, including $\mathrm{Nb}[10,12], \mathrm{In}, \mathrm{Ge}[11]$ and $\mathrm{Al}[13]$. The general trend is that increasing the content of the sixth element (frequently of larger radius) changes 
the FCC structure to amorphous, or BCC in the case of Al. It was suggested that the larger size of Al made it effectively an impurity in a matrix that could be better accommodated by the lower coordination of the BCC lattice [13]. It has also been suggested that momentum transfer from the back-reflected Ar atoms in the sputtering process for the $\mathrm{Nb}$ alloy films caused an increase in crystallinity by reducing micro-stress [12].

Pulsed laser deposition (PLD) is a widely used method for the deposition of thin films, particularly compounds and alloys. Among its attractions are that it has lesser problems with incongruent evaporation than some methods and it is equally applicable to low vapour pressure materials as high. It is distinct in nature from sputter-deposition in that the instantaneous deposition rate may be much higher and the energy of the arriving species can be much higher. It is known that the properties of HEA are strongly dependent on manufacturing conditions such a slowly changing temperature, or quench cooling. Thin film deposition is by nature quite different from the creation of bulk materials enabling the creation of materials with differing properties and the use of PLD would open-up a new parameter space for the controlling the deposition of HEA films. For this reason, an investigation into the deposition of HEA films by PLD has been instigated and is reported here.

In this paper, the deposition of thin films of AICrFeCoNiCu by PLD is reported and the influence on structure and composition of the deposition temperature discussed. The potentially important incidence of surface segregation within the alloy is also reported. 


\section{Experimental Methods}

The HEA depositions were performed using PLD onto borosilicate glass. The resulting thin films were then analysed using symmetric X-ray diffraction (XRD) and X-ray photoelectron spectroscopy (XPS) to investigate for crystal structure and composition.

The equimolar target for PLD was manufactured by combining the appropriate amounts of each element in an argon arc furnace with a tungsten electrode and a copper hearth. The slug of alloy was melted repeatedly to ensure good mixing of the elements. The resulting slug was cut to shape using spark erosion and the front face was polished. Before use for deposition the target surface was cleaned in situ using a low laser fluence.

The Loughborough PLD system $[18,19]$ is based on a design by IWS Dresden [20] and has a base pressure of $2 \times 10^{-10}$ mbar. Deposition uses a pulsed and frequencydoubled Quanta Ray Nd:YAG laser operating at $10 \mathrm{~Hz}$ and wavelength $532 \mathrm{~nm}$. Residual fundamental infra-red radiation is rejected by two wavelength-selective dichroic mirrors and a lens is used to focus the beam into the chamber through a fused silica viewport.

The films were deposited onto $10 \times 10 \mathrm{~mm}$ borosilicate glass squares (D263 Menzel Gläser) supplied by Agar Scientific Ltd. The substrates were cleaned ex situ using isopropyl alcohol and acetone and were heated under high-vacuum in the load-lock at $120{ }^{\circ} \mathrm{C}$ for four hours before transfer into the deposition chamber. Further outgassing was carried out at $300^{\circ} \mathrm{C}$ until the chamber pressure had fallen to $10^{-8}$ 
mbar before the substrates were allowed to cool in vacuum to the desired deposition temperature. The thin films were deposited in vacuum using a fluence of $2.0 \mathrm{Jcm}^{-2}$ and the mean deposition rate in was $0.25 \mathrm{~nm} \mathrm{~min}^{-1}$. The film thickness was determined by grazing incidence $\mathrm{X}$-ray reflectivity (XRR) using a specially modified Bruker D5000 and fitted using GenX [21].

Structural analysis was performed using XRD in the Bragg-Brentano $\theta-2 \theta$ geometry symmetrically about the surface normal utilising a Bruker D2 Phaser and the filtered Cu Ka line at $0.154 \mathrm{~nm}$. A modified Bruker D5000 was also employed to obtain a GIXRD $\theta-2 \theta$ scan to confirm the degree of texture. Compositional analysis of the target was performed using EDS on a Hitachi TM3030 and compositional analysis and depth profiles of selected thin films were obtained by XPS using a Thermo KAlpha employing an X-ray energy of $1486.7 \mathrm{eV}$. All the equipment is located in the laboratories of Loughborough University, the TM3030 and Thermo K-Alpha are operated by Loughborough Materials Characterisation Centre and the remaining equipment is based in the Department of Physics.

\section{Results and Discussion}

An off-cut of the target was examined for composition and structure using EDS (several different areas were examined) and XRD, the results being shown in Figure

1. The composition of the target (Figure 1(a)) is close to equimolar, as expected, being Cu:Ni:Co:Fe:Cr:Al 15.2\%:17.1\%:18.4\%:18.1\%:17.1\%:14.1\%. A similar underrepresentation of $\mathrm{Al}$ in EDS from this material has been seen previously and ascribed to higher absorption [22]. A $\theta-2 \theta$ XRD scan of the target is shown in Figure 
1(b). As is common for this material [22], only FCC and BCC phases are present, the FCC reflections being indicated in the figure by $\bullet$ and the BCC by $\boldsymbol{\square}$. The FCC(111) reflection is much stronger than that of the $\mathrm{BCC}(110)$, as may be expected from the structure factors and multiplicities. However, the ratio of the peak areas is just over 5:1 indicating that FCC is slightly favoured, something more usually associated with copper rich alloys. Electron microscopy of the target indicates the presence of some small copper rich areas that could possibly be the cause of this (though of area fraction closer to just a few percent rather than 20\%). Given that the EDS was taken over several sample points and the size of the PLD laser spot is macroscopic ( 1 $\mathrm{mm}$ ) any phase separation will not affect the resulting film composition. A report on nano-structured materials suggests that both FCC and BCC phases are observed in equimolar alloys, but that much higher Al content results in BCC being dominant [23], whereas FCC is dominant with lower Al content.

To investigate the structure of the HEA films and the effect of depositing at elevated substrate temperatures, a series of films was deposited at temperatures between room temperature $(\mathrm{RT})$ and $300^{\circ} \mathrm{C}$. Figure $2(\mathrm{a})$ shows the $\theta-2 \theta$ X-ray diffraction scan collected symmetrically about the surface normal for a $35 \mathrm{~nm}$ thin film deposited at room temperature onto glass. Only two reflections are evident; the FCC (111) at $43.2^{\circ}$ and the BCC (110) at $44.5^{\circ}$. This implies the existence of at least two types of domain, each with an orientational texture such that the most densely occupied plane is parallel to the growth plane, as is common in thin films. It should also be noted that unlike the bulk material measured in Figure 1(b), the FCC (111) reflection is significantly broader than the BCC (110) implying that the FCC crystallites are smaller than the BCC. That no other significant orientations of crystallites are present 
is confirmed by an out-of-plane grazing incidence $\theta-2 \theta$ X-ray diffraction scan shown in Figure 2(b). This scan shows only the BCC (110) reflection at $2 \theta$ around $45^{\circ}$ and the FCC (220) reflection at $2 \theta$ around $74^{\circ}$, each being allowed due to the mosaicity of the film and the fact that these planes have an angle from their respective oriented crystal normal of about half the $2 \theta$ angle of reflection. Figure 2(c) shows an electron micrograph of the droplets on the film caused by "splashing".

The presence of the FCC (111) and BCC (110) texturing has been observed previously in thin films of this and similar materials, though usually in considerably thicker films [11-16]. In a study of thin films of AlCoCrCuFeNi deposited onto silicon by room temperature sputter deposition from mosaic targets [14], the films were found to texture in FCC (111) for lower Al content and in both FCC (111) and BCC (110) with slightly higher content. Subsequent annealing to temperatures above $300^{\circ} \mathrm{C}$ resulted in either reaction with substrate or segregation into binary alloys. In another study of deposition by sputter deposition from compressed powder targets [13] CoCrCuFeNi targets were found to texture in the FCC (111) but addition of Al introduced BCC (110) texture. It was suggested that the lower coordination of the BCC structure could accommodate the larger Al atoms more easily.

A series of $\theta-2 \theta$ X-ray diffraction scans in the region of the two reflections taken from $35 \mathrm{~nm}$ thin films deposited at onto substrates at temperatures between room temperature and $300^{\circ} \mathrm{C}$ is shown in Figure 3 . The data are represented by points and the results of peak fitting using a pseudo-voigt function are represented by solid lines. The results of this peak-fitting averaged across all samples gives cubic lattice parameters of $3.61 \AA$ for $\mathrm{FCC}$ and $2.87 \AA$ for BCC. These are consistent with 
previous work on as-cast and quench-cooled bulk alloys of the same material [22]. It can be seen that with increasing deposition temperature, the uniaxial texture falls to being effectively zero at $300^{\circ} \mathrm{C}$. It should be noted that in PLD the energy of the arriving species exceeds that in magnetron sputtering by around a factor of ten [24] and in the magnetron study there was evidence that momentum enhances crystallinity by densification.

The peaks shown in Figure 3 were fitted using a pseudo-voigt function and Figure 4 shows the peak areas determined. The values of the areas for FCC (111) compared with BCC (110) are twice as large as may be expected from the structure factor, suggesting that the volume of FCC crystallites exceeds that of BCC, despite the FCC crystals being smaller as suggested by the peak width. Using the Scherrer method [25] the peak width for FCC gives a crystal size of around the same as the film thickness, as could be expected. The crystal size from the BCC (110) is about twice this. The reason for this is not clear, however a complication of PLD compared with other deposition techniques is that the growing film is also splashed with droplets [26], so we need to be aware that the observed reflections may also be associated with these larger pieces of material, but the droplets are unlikely to have a special texture. Figure 4 also shows the peak area ratio, which indicates that as the deposition temperature increases, the FCC crystallites with preferred orientational texture persist longer than the BCC crystallites with preferred orientational texture.

The composition of the films was investigated using XPS ex-situ. XPS samples only the surface region due to the short electron attenuation length at these energies [27], while in some cases EDS could be more valuable for average compositions as the technique samples all the film. However, EDS would be not appropriate here as the 
large penetration and escape depth mean it samples many elements within the glass substrate. Depth profiling into the film by sputter erosion would enable all the film to be analysed by XPS, but the difference in sputter yields between elements would result in an apparent composition that was altered from its original [28].

For the film deposited onto glass at room temperature, Figure 5 shows survey scans and details of specific emission peaks both before and after etching with an $\mathrm{Ar}^{+}$ beam; several features are apparent. The survey scans (Figure 5(a) and (h)) show only the six principal elements plus oxygen and carbon, which originate from ex situ contamination. Following etching the carbon and oxygen are mostly removed and their residual is most likely from the background vacuum given the time taken to record the core level scans.

In Figure 5, from the detailed scans of peaks originating from the six metals, it can be seen that before etching the metals show clear signs of oxidation, with the exception of $\mathrm{Cu}$ and perhaps $\mathrm{Ni}$. This is indicated by a higher binding energy peak that is clearly visible compared with the typically metallic spectra shown after sputtering. It is known that the $\mathrm{Cu} 2 \mathrm{p}$ transitions are relatively insensitive to low level of oxidation [29], so this may not indicate a lack of oxidation. The Al 2s peak in Figure 5(g) in particular is dominated by the oxide peak $1.5 \mathrm{eV}$ higher in binding energy than the metallic peak [30]. The $\mathrm{Cr} 2 \mathrm{p}_{3 / 2}$ also shows a dominant oxide peak 3 eV higher in binding energy than the metallic peak [31]. Of course, both elements are noted for ready oxidation.

The detailed scans of the core levels are difficult to quantify due to the large number of elements present that result in overlapping peaks, both photoemission and those 
due to Auger transitions; this is particularly true for Fe and Al. Indeed, the Al 2s was selected as the $2 p$ overlaps with the $\mathrm{Cu} 3 p$. The transitions that were used later for depth profiling are marked with * and obvious peak interactions are marked with + in the figure.

The variation of the composition with depth of the films deposited at room temperature, $150^{\circ} \mathrm{C}$ and $300^{\circ} \mathrm{C}$ was investigated by depth profiling using $500 \mathrm{eV} \mathrm{Ar}^{+}$ ions. This is illustrated in Figure 6; panels (a)-(c) show the profiles of the six principal elements plus $\mathrm{O}$ and $\mathrm{C}$, while panels $(\mathrm{d})-(\mathrm{f})$ show the same profiles re-calculated to show atomic percentage considering the metallic elements only. The x-axes are the etch time in seconds and a nominal depth based on the known etch rate for $\mathrm{Ta}_{2} \mathrm{O}_{5}$. The depth profiles are determined by collecting a series of "snap" spectra around the core-levels and the area of one peak is determined by integrating above a Shirley background [32]. Given the problem of overlapping peaks mentioned earlier these profiles are indicative of concentration variation with depth but may not be completely accurate in terms of absolute atomic concentration, however they should give a reasonable picture of the variation of composition with conditions.

Several things are apparent from the profiles. The first of these is that the oxygen and carbon contamination are localised at the surface, as should be expected. The persistence of the oxygen in the profile is most likely due to continuing low-level contamination from the residual vacuum onto some quite reactive metals. The depth of the oxygen contamination exceeds that of the carbon suggesting a layer of metal oxide to about $3 \mathrm{~nm}$. 
In the bulk of the room-temperature deposited film (Figure 6(d)) the apparent atomic concentrations are similar, given the caveats on the peak interaction, suggesting reasonably close to equi-atomic concentration. The element with the lowest atomic concentration, $\mathrm{Fe}$, is also the one that is most difficult to distinguish accurately so the values for this should be treated with some caution. The elements $\mathrm{Ni}$ and Co seem to be slightly over-represented whereas the others are similar. Ways in which the mechanisms of PLD may affect stoichiometry include in the ablation from the target [33], the behaviour of the plume [34] and in re-sputtering from the film [33,35]. The latter may be important here as in vacuum the plume is not decelerated, and hence very energetic species are impinging onto the growing film.

For the films deposited at $150^{\circ} \mathrm{C}$ and $300^{\circ} \mathrm{C}$ (Figure 6 (e) and (f) respectively) the scatter in concentration between elements increases and, in particular, the amount of Al falls between room temperature and $150^{\circ} \mathrm{C}$ and the amount of Cu falls between $150^{\circ} \mathrm{C}$ and $300^{\circ} \mathrm{C}$. When considering these compositions, we should be aware that they are normalised to $100 \%$ across all six elements, so a depletion of one element will lead to an apparent enhancement of the others.

The elements $\mathrm{Al}$ and $\mathrm{Cu}$ are known to be problematic for diffusion, but this is quite a low temperature. Reports indicate that the diffusivity of $\mathrm{Cu}$ in glass at $300^{\circ} \mathrm{C}$ is low [36] and the diffusion of Al is not high [37] and should be limited by the presence of aluminium oxide at the interface. However, given the short distances involved at comparatively long times it may be that the diffusion at $300^{\circ} \mathrm{C}$ is sufficient to explain the loss $[38,39]$. The other possible mechanisms for loss of the elements would be diffusion across the substrate onto the holder (unlikely given the distances involved) 
re-evaporation (also unlikely), or re-sputtering due to the energetic plume impacting on the film.

Returning to the discussion of the crystallinity of the films, it is interesting to note that a previous study [22] associated the presence of FCC structure within the material with the presence of copper precipitates, copper having the highest positive enthalpy of mixing with the other transition metals in the alloy. It has also been suggested that the larger size of Al stabilises the BCC structure, which has lower coordination number [13]. Thus, when the XRD data are cross-compared with the XPS concentrations it may be tempting to associate the fall in the crystallinity to the reduced concentration of these two elements with deposition temperature, however it must be less straight-forward than this as the concentrations of these elements do not map directly to the XRD peak areas. The study of bulk alloys indicated a complex phase separation in as-cast alloys [22] and the variety of competing processes induced by PLD growth will make the situation, if anything, more complex than bulk preparation.

The depth profiles in Figure 6 also show some detail of the atomic concentrations in the first $5 \mathrm{~nm}$, where surface segregation can be seen for the elements $\mathrm{Al}$ and $\mathrm{Cr}$. Although the concentration of all metallic elements is lower in the surface region, due primarily to oxidation, the concentrations of $\mathrm{Al}$ and $\mathrm{Cr}$ do not fall as much as the others. This is more easily observable in Figure 6(d-f) in which the $\mathrm{O}$ and $\mathrm{C}$ contributions have been removed. The surface concentration of Al is strongly enhanced relative to the other element, while that of $\mathrm{Cr}$ is slightly enhanced. This issue may be of concern in applications where high-entropy alloys are used for surface-related properties, changing their contact or anti-corrosion properties. 
The existence of surface segregation in alloys, particularly dilute binary alloys, is long known [40]. Several driving mechanisms for this have been proposed including surface energy [41], atomic size [42] and adsorbate induced segregation [43]. The segregation of Al may be expected on several grounds. The surface energy of $\mathrm{Al}$ is lower than that of other elements in the alloy [44] so simple thermodynamic grounds may predict this. In addition, Al atoms are larger than the other elements which also may favour their segregation to the surface to relieve lattice strain [42]. It has also been shown that the presence of $\mathrm{O}$ on the surface can induce the segregation of $\mathrm{Al}$ from dilute alloys with $\mathrm{Cu}$ [43]. The segregation of $\mathrm{Cr}$ is less obvious, but here it may also be driven by oxidation and the segregation of $\mathrm{Cr}$ from $\mathrm{Fe}-\mathrm{Cr}$ alloys is well known [45]. The apparent subsurface enhancement of $\mathrm{Cu}$ is interesting, but care needs to be taken here; this may be an artefact caused by the sputter yield of $\mathrm{Cu}$ being higher than the other elements [46] resulting in an altered layer.

\section{Summary}

Thin films of AlCrFeCoNiCu have been prepared by PLD. Films deposited at room temperature exhibit a mix of $\mathrm{FCC}$ and $\mathrm{BCC}$ structures. Both crystal structures reduce with increasing deposition temperature, the fall in $\mathrm{BCC}$ commencing at lower temperature than the FCC associated with reduction of the content of $\mathrm{Al}$ and $\mathrm{Cu}$. XPS depth profiles show that the surface of the HEA films has higher concentration of $\mathrm{Al}$ and $\mathrm{Cr}$.

\section{Acknowledgments}


This research did not receive any specific grant from funding agencies in the public, commercial, or not-for-profit sectors. The author acknowledges the use of the facilities and the assistance of Pat Cropper in the Loughborough Materials Characterisation Centre. Laura Stuffins assisted with the X-ray reflectivity measurements and the author would also like to acknowledge the help and encouragement of Andrew Caruana, Chris Cox and Kelly Morrison.

\section{References}

[1] M.C. Gao, J.W. Yeh, P.K. Liaw, High-entropy alloys : fundamentals and applications, Springer, Switzerland, 2016.

[2] J.-W. Yeh, S.-K. Chen, S.-J. Lin, J.-Y. Gan, T.-S. Chin, T.-T. Shun, C.-H. Tsau, S.-Y. Chang, Nanostructured High-Entropy Alloys with Multiple Principal Elements: Novel Alloy Design Concepts and Outcomes, Adv. Eng. Mater. 6 (2004) 299-303. doi:10.1002/adem.200300567.

[3] B. Cantor, I.T.H. Chang, P. Knight, A.J.B. Vincent, Microstructural development in equiatomic multicomponent alloys, Mater. Sci. Eng. A. 375377 (2004) 213-218. doi:10.1016/j.msea.2003.10.257.

[4] X.B. Feng, W. Fu, J.Y. Zhang, J.T. Zhao, J. Li, K. Wu, G. Liu, J. Sun, Effects of nanotwins on the mechanical properties of $\mathrm{Al} \times \mathrm{CoCrFeNi}$ high entropy alloy thin films, Scr. Mater. 139 (2017) 71-76. doi:10.1016/j.scriptamat.2017.06.009.

[5] D. Dou, X.C. Li, Z.Y. Zheng, J.C. Li, Coatings of FeAlCoCuNiV high entropy 
alloy, Surf. Eng. 32 (2016) 766-770. doi:10.1080/02670844.2016.1148380.

[6] X. Feng, G. Tang, M. Sun, X. Ma, L. Wang, K. Yukimura, Structure and properties of multi-targets magnetron sputtered ZrNbTaTiW multi-elements alloy thin films, Surf. Coatings Technol. 228 (2013) S424-S427. doi:10.1016/j.surfcoat.2012.05.038.

[7] A. Kauffmann, M. Stüber, H. Leiste, S. Ulrich, S. Schlabach, D.V. Szabó, S. Seils, B. Gorr, H. Chen, H.-J. Seifert, M. Heilmaier, Combinatorial exploration of the High Entropy Alloy System Co-Cr-Fe-Mn-Ni, Surf. Coatings Technol. 325 (2017) 174-180. doi:10.1016/j.surfcoat.2017.06.041.

[8] R.-C. Lin, T.-K. Lee, D.-H. Wu, Y.-C. Lee, A Study of Thin Film Resistors Prepared Using Ni-Cr-Si-Al-Ta High Entropy Alloy, Adv. Mater. Sci. Eng. 2015 (2015) 1-7. doi:10.1155/2015/847191.

[9] P.-C. Lin, C.-Y. Cheng, J.-W. Yeh, T.-S. Chin, Soft Magnetic Properties of High-Entropy Fe-Co-Ni-Cr-Al-Si Thin Films, Entropy. 18 (2016) 308. doi:10.3390/e18080308.

[10] B.R. Braeckman, F. Misják, G. Radnóczi, M. Caplovicová, P. Djemia, F. Tétard, L. Belliard, D. Depla, The nanostructure and mechanical properties of nanocomposite Nb x -CoCrCuFeNi thin films, Scr. Mater. 139 (2017) 155-158. doi:10.1016/j.scriptamat.2017.06.046.

[11] B.R. Braeckman, F. Misják, G. Radnóczi, D. Depla, The influence of Ge and In 
addition on the phase formation of CoCrCuFeNi high-entropy alloy thin films, Thin Solid Films. 616 (2016) 703-710. doi:10.1016/j.tsf.2016.09.021.

[12] B.R. Braeckman, D. Depla, Structure formation and properties of sputter deposited $\mathrm{Nb}$ x-CoCrCuFeNi high entropy alloy thin films, J. Alloys Compd. 646 (2015) 810-815. doi:10.1016/j.jallcom.2015.06.097.

[13] B.R. Braeckman, F. Boydens, H. Hidalgo, P. Dutheil, M. Jullien, A.-L. Thomann, D. Depla, High entropy alloy thin films deposited by magnetron sputtering of powder targets, Thin Solid Films. 580 (2015) 71-76. doi:10.1016/j.tsf.2015.02.070.

[14] V. Dolique, A.-L. Thomann, P. Brault, Y. Tessier, P. Gillon, Thermal stability of AlCoCrCuFeNi high entropy alloy thin films studied by in-situ XRD analysis, Surf. Coatings Technol. 204 (2010) 1989-1992. doi:10.1016/j.surfcoat.2009.12.006.

[15] V. Dolique, A.-L. Thomann, P. Brault, Y. Tessier, P. Gillon, Complex structure/composition relationship in thin films of AlCoCrCuFeNi high entropy alloy, Mater. Chem. Phys. 117 (2009) 142-147. doi:10.1016/j.matchemphys.2009.05.025.

[16] B.R. Braeckman, P. Djemia, F. Tétard, L. Belliard, D. Depla, Impuritycontrolled film growth and elastic properties of CoCrCuFeNi thin films, Surf. Coatings Technol. 315 (2017) 475-483. doi:10.1016/j.surfcoat.2017.03.014. 
[17] Z. An, H. Jia, Y. Wu, P.D. Rack, A.D. Patchen, Y. Liu, Y. Ren, N. Li, P.K. Liaw, Solid-Solution CrCoCuFeNi High-Entropy Alloy Thin Films Synthesized by Sputter Deposition, Mater. Res. Lett. 3 (2015) 203-209. doi:10.1080/21663831.2015.1048904.

[18] M.S. Al-Busaidy, O.E. Kusmartseva, M.D. Crapper, Pulsed laser deposition of metallic multilayers: The influence of laser power on microstructure, in: Appl. Phys. A Mater. Sci. Process., 2004: pp. 1453-1456. doi:10.1007/s00339-0042809-5.

[19] A.J. Caruana, M.D. Cropper, Orientationally textured thin films of WOx deposited by pulsed laser deposition, Mater. Res. Express. 1 (2014) 46408. doi:10.1088/2053-1591/1/4/046408.

[20] R. Dietsch, T. Holz, H. Mai, C.-F. Meyer, R. Scholz, B. Wehner, High precision large area PLD of X-ray optical multilayers, Appl. Surf. Sci. 127-129 (1998) 451-456. doi:10.1016/S0169-4332(97)00671-5.

[21] M. Björck, G. Andersson, GenX: An extensible X-ray reflectivity refinement program utilizing differential evolution, J. Appl. Crystallogr. 40 (2007) 11741178. doi:10.1107/S0021889807045086.

[22] S. Singh, N. Wanderka, B.S. Murty, U. Glatzel, J. Banhart, Decomposition in multi-component AICoCrCuFeNi high-entropy alloy, Acta Mater. 59 (2011) 182-190. doi:10.1016/j.actamat.2010.09.023. 
[23] J.W. Yeh, S.K. Chen, S.J. Lin, J.Y. Gan, T.S. Chin, T.T. Shun, C.H. Tsau, S.Y. Chang, Nanostructured high-entropy alloys with multiple principal elements: Novel alloy design concepts and outcomes, Adv. Eng. Mater. 6 (2004) 299_ 303+274. doi:10.1002/adem.200300567.

[24] B. Shin, J.P. Leonard, J.W. McCamy, M.J. Aziz, Comparison of morphology evolution of $\mathrm{Ge}(001)$ homoepitaxial films grown by pulsed laser deposition and molecular-beam epitaxy, Appl. Phys. Lett. 87 (2005) 181916. doi:10.1063/1.2108115.

[25] A.L. Patterson, The Scherrer Formula for X-Ray Particle Size Determination, Phys. Rev. 56 (1939) 978-982. doi:doi.org/10.1103/PhysRev.56.978.

[26] J.C.S. Kools, Pulsed Laser Deposition of Metals, in: D.B. Chrisey, G.K. Hubler (Eds.), Pulsed Laser Depos. Thin Film., 1994: pp. 455-471.

[27] C.J. Powell, The quest for universal curves to describe the surface sensitivity of electron spectroscopies, J. Electron Spectros. Relat. Phenomena. 47 (1988) 197-214. doi:10.1016/0368-2048(88)85012-6.

[28] S. Hofmann, Y.S. Han, J.Y. Wang, Depth resolution and preferential sputtering in depth profiling of sharp interfaces, Appl. Surf. Sci. 410 (2017) 354-362. doi:10.1016/j.apsusc.2017.03.110.

[29] M.C. Biesinger, Advanced analysis of copper X-ray photoelectron spectra, Surf. Interface Anal. 49 (2017) 1325-1334. doi:10.1002/sia.6239. 
[30] Y. Wu, E. Garfunkel, T.E. Madey, Growth and oxidation of ultra-thin Al films on the Re (0001) surface, Surf. Sci. 365 (1996) 337-352. doi:10.1016/00396028(96)00699-1.

[31] M.C. Biesinger, B.P. Payne, A.P. Grosvenor, L.W.M. Lau, A.R. Gerson, R.S.C. Smart, Resolving surface chemical states in XPS analysis of first row transition metals, oxides and hydroxides: Cr, Mn, Fe, Co and Ni, Appl. Surf. Sci. 257 (2011) 2717-2730. doi:10.1016/j.apsusc.2010.10.051.

[32] D.A. Shirley, High-Resolution X-Ray Photoemission Spectrum of the Valence Bands of Gold, Phys. Rev. B. 5 (1972) 4709-4714. doi:10.1103/PhysRevB.5.4709.

[33] E. van de Riet, J.C.S. Kools, J. Dieleman, Incongruent transfer in laser deposition of FeSiGaRu thin films, J. Appl. Phys. 73 (1993) 8290-8296. doi:10.1063/1.353447.

[34] C.B. Arnold, M.J. Aziz, Stoichiometry issues in pulsed-laser deposition of alloys grown from multicomponent targets, Appl. Phys. A Mater. Sci. Process. 69 (1999) S23-S27. doi:10.1007/s003399900182.

[35] T. Scharf, H.U. Krebs, Influence of inert gas pressure on deposition rate during pulsed laser deposition, Appl. Phys. A Mater. Sci. Process. 75 (2002) 551-554. doi:10.1007/s00339-002-1442-4.

[36] J.D. McBrayer, Diffusion of Metals in Silicon Dioxide, J. Electrochem. Soc. 133 
(1986) 1242. doi:10.1149/1.2108827.

[37] H.G. Francois-Saint-Cyr, F.A. Stevie, J.M. McKinley, K. Elshot, L. Chow, K.A. Richardson, Diffusion of 18 elements implanted into thermally grown SiO[sub 2], J. Appl. Phys. 94 (2003) 7433. doi:10.1063/1.1624487.

[38] D. Gupta, K. Vieregge, K. V. Srikrishnan, Copper diffusion in amorphous thin films of $4 \%$ phosphorus-silcate glass and hydrogenated silicon nitride, Appl. Phys. Lett. 61 (1992) 2178-2180. doi:10.1063/1.108287.

[39] X. Qingfeng, G. Sasaki, H. Fukunaga, Interfacial microstructure of anodicbonded Al/glass, J. Mater. Sci. Mater. Electron. 13 (2002) 83-88. doi:10.1023/A:1013650123007.

[40] J.P. Muscat, Surface segregation in binary alloys: a simple theoretical model, J. Phys. C Solid State Phys. 15 (1982) 867-879. doi:10.1088/00223719/15/4/030.

[41] S.H. Overbury, P.A. Bertrand, G.A. Somorjai, Surface composition of binary systems. Prediction of surface phase diagrams of solid solutions, Chem. Rev. 75 (1975) 547-560. doi:10.1021/cr60297a001.

[42] F.F. Abraham, T. Nan-Hsiung, G.M. Pound, Bond and strain energy effects in surface segregation: An atomic calculation, Surf. Sci. 83 (1979) 406-422. doi:10.1016/0039-6028(79)90053-0. 
[43] T. Kravchuk, A. Hoffman, Oxygen induced segregation of aluminum to a-Cu$\mathrm{Al}(100)$ alloy surfaces studied by low energy ion scattering and X-ray photoelectron spectroscopy, Surf. Sci. 601 (2007) 87-94. doi:10.1016/j.susc.2006.09.008.

[44] A. Miedema, Surface Energies of Solid Metals, Zeitschrift Fur Met. 69 (1978) 287-292.

[45] P.A. Dowben, M. Grunze, D. Wright, Surface segregation of chromium in a Fe72Cr28(110) crystal, Surf. Sci. 134 (1983) L524-L528. doi:10.1016/00396028(83)90424-7.

[46] National Physical Laboratory, Sputter Yield Values, (2014). http://www.npl.co.uk/science-technology/surface-andnanoanalysis/services/sputter-yield-values (accessed January 1, 2018). 


\section{List of Figures}

Figure 1: (a) the composition of the target and a $35 \mathrm{~nm}$ film deposited onto a silicon substrate as determined by EDS. (b) $\theta-2 \theta$ X-ray diffraction of the target with the expected positions of reflections due to FCC and BCC structures shown.

Figure 2: X-ray diffraction of a thin film deposited onto glass at room temperature. (a) a $\theta-2 \theta$ scan collected symmetrically about the surface normal, showing both FCC and BCC crystallites with preferred orientations of (111) and (110) respectively. (b) a grazing incidence $\left(1^{\circ}\right) \theta-2 \theta$ scan showing BCC (110) and FCC (220) reflections. (c) A low-resolution SEM image showing the droplet distribution on the thin film surface.

Figure 3: $\theta-2 \theta$ scans collected symmetrically about the surface normal of a thin film deposited onto glass at various temperatures, showing the detail of the FCC (111) and BCC (110) reflections together with peak fits as detailed in the text.

Figure 4: The relative peak areas for the FCC (111) and BCC (110) reflections from thin films deposited onto glass at various temperatures.

Figure 5: X-ray photoelectron spectra of a $\mathrm{AlCrFeCoNiCu}$ deposited onto glass at room temperature before and after etching using a $500 \mathrm{eV} \mathrm{Ar}^{+}$beam. (a) survey scan of the as-deposited film. (b-g) high resolution scans of the principal elements before (black line) and after the etch (grey line). (h) the survey scan after the etch.

Figure 6: X-ray photoelectron depth profiles of the films showing and omitting carbon and oxygen contributions for films deposited at (a\&d) room temperature, (b\&e) $150^{\circ} \mathrm{C}$, and (c\&f) $300^{\circ} \mathrm{C}$, 

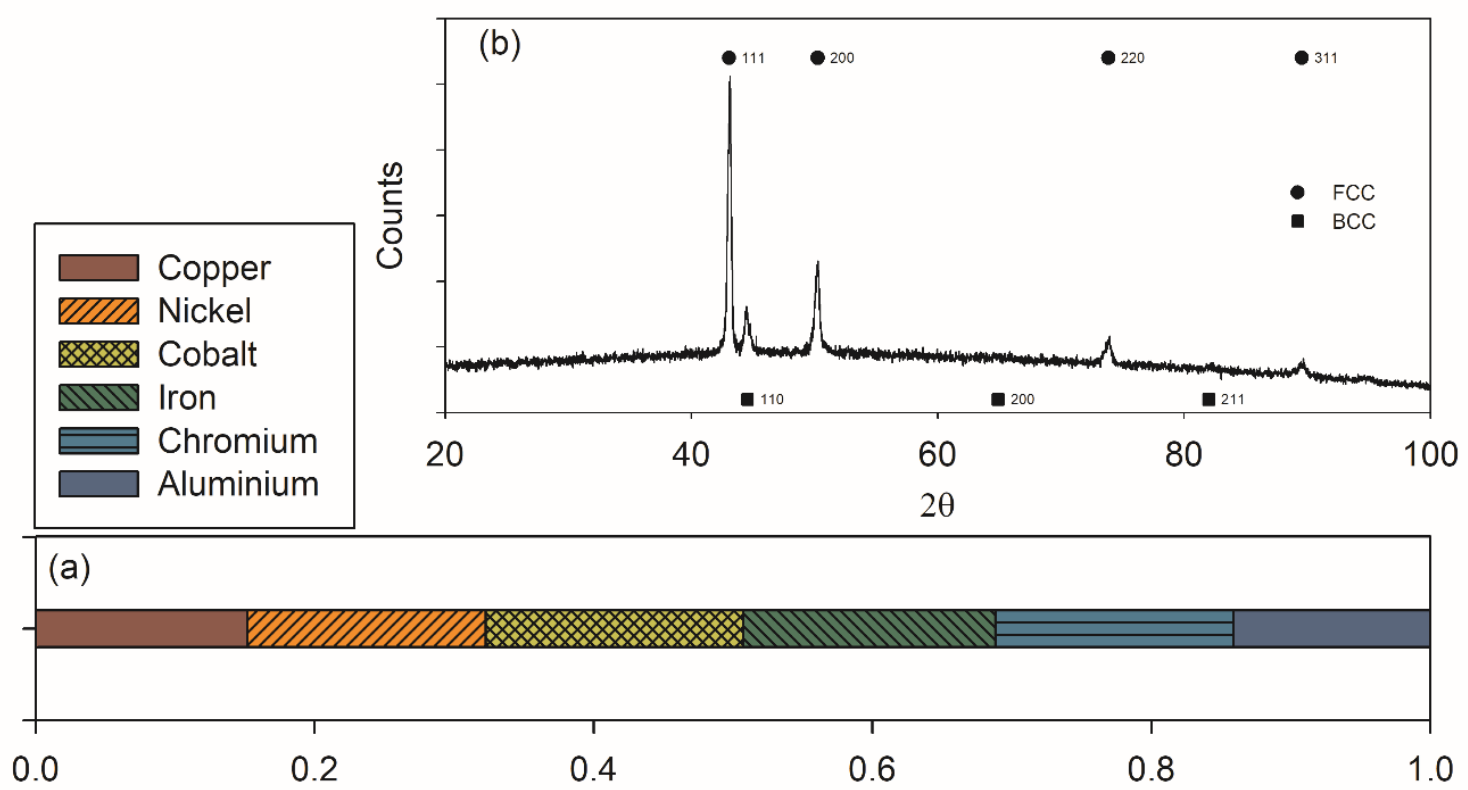

PLD Target Atomic Concentration from EDS

Figure 1 


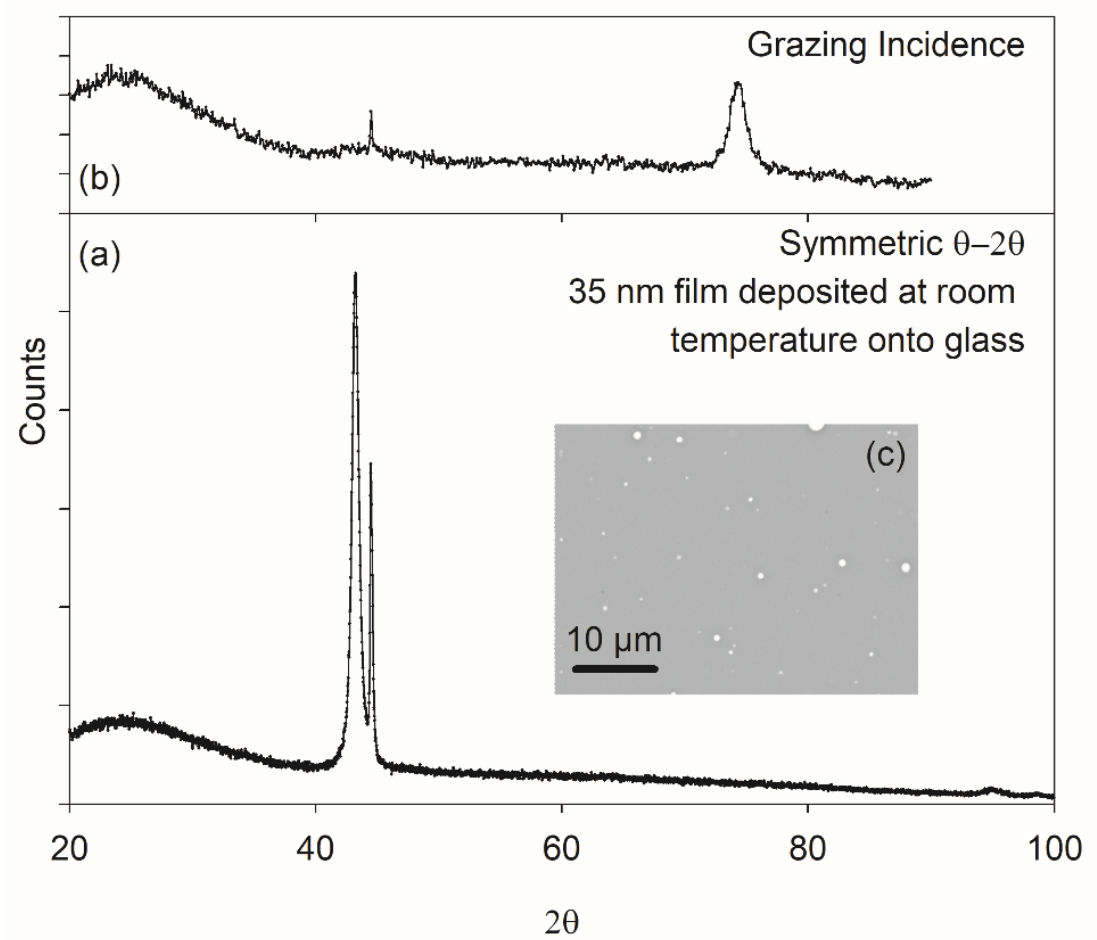

Figure 2 


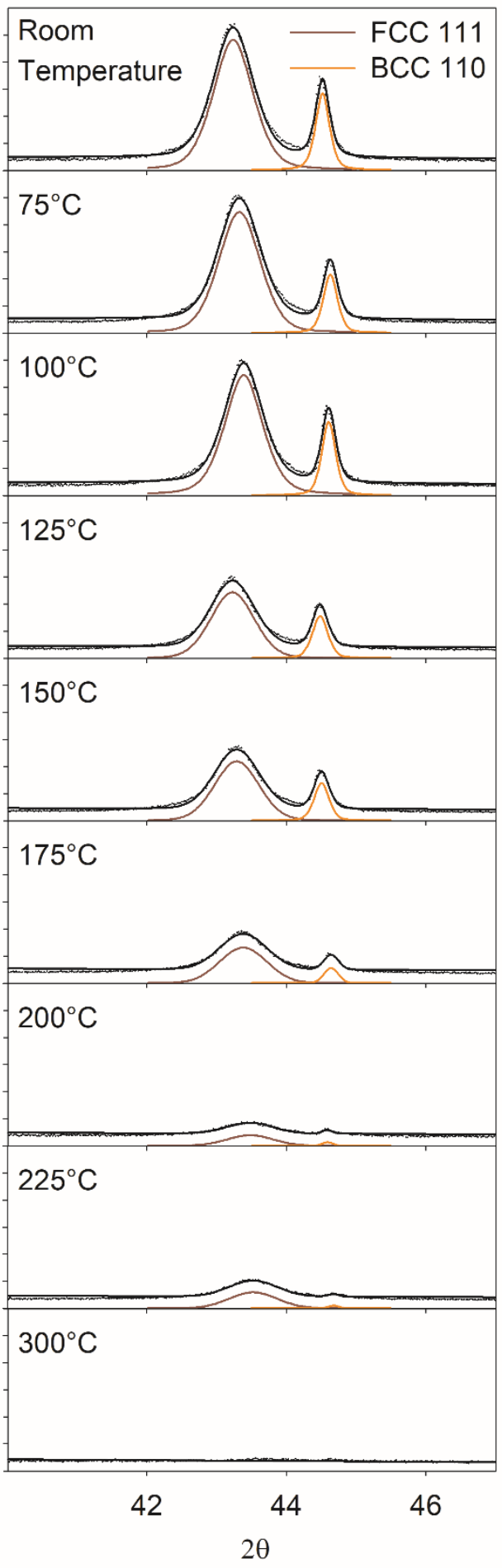

Figure 3 


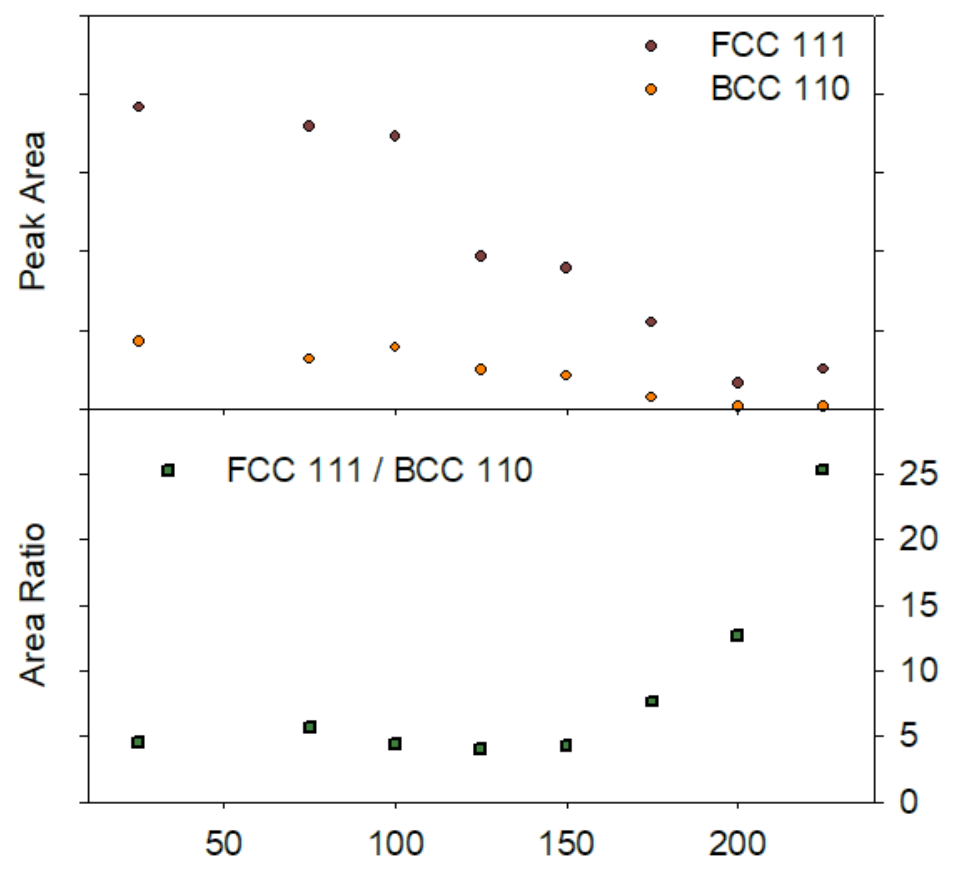

Deposition Substrate Temperature $\left({ }^{\circ} \mathrm{C}\right)$

Figure 4 


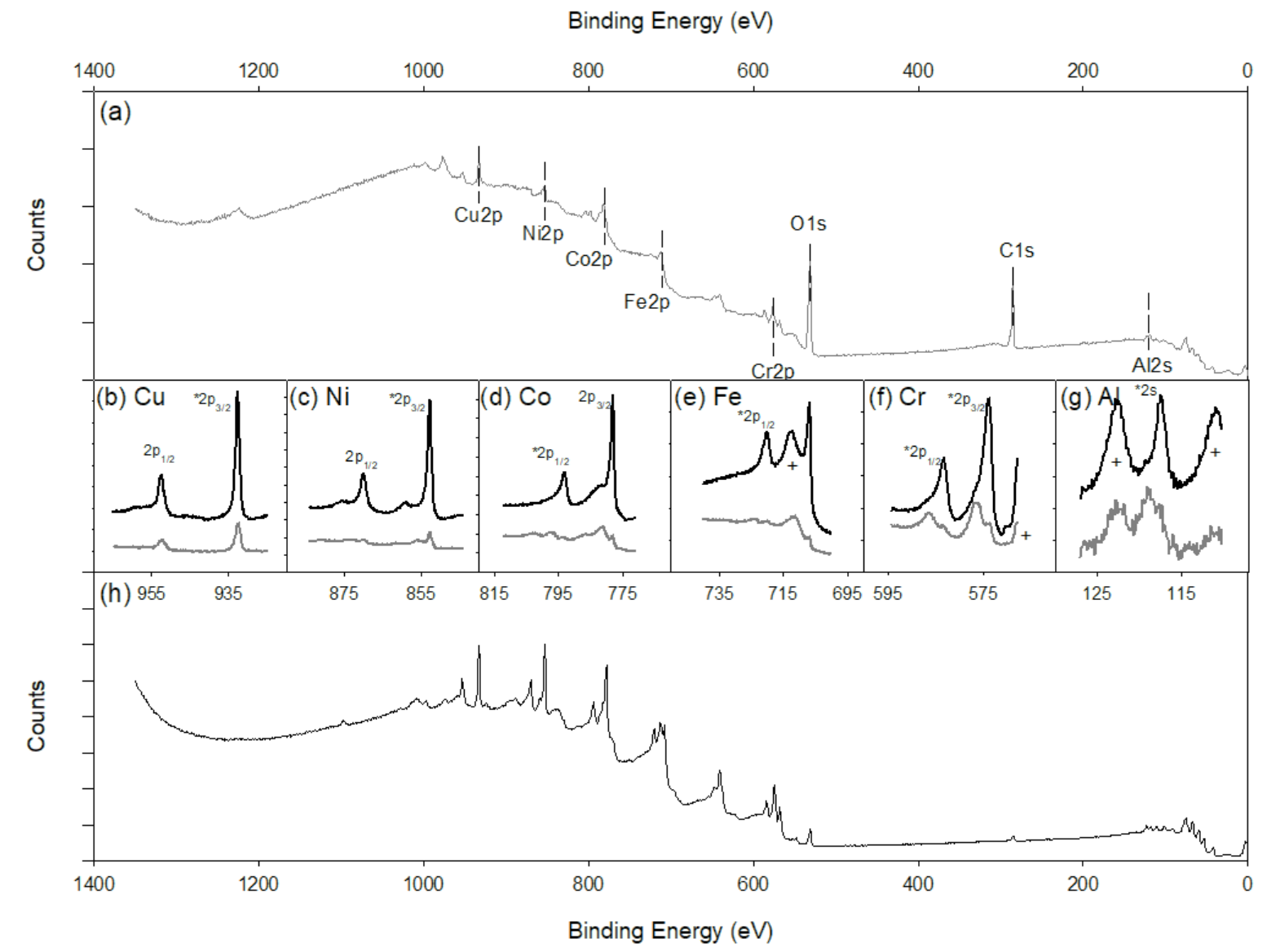

Figure 5 


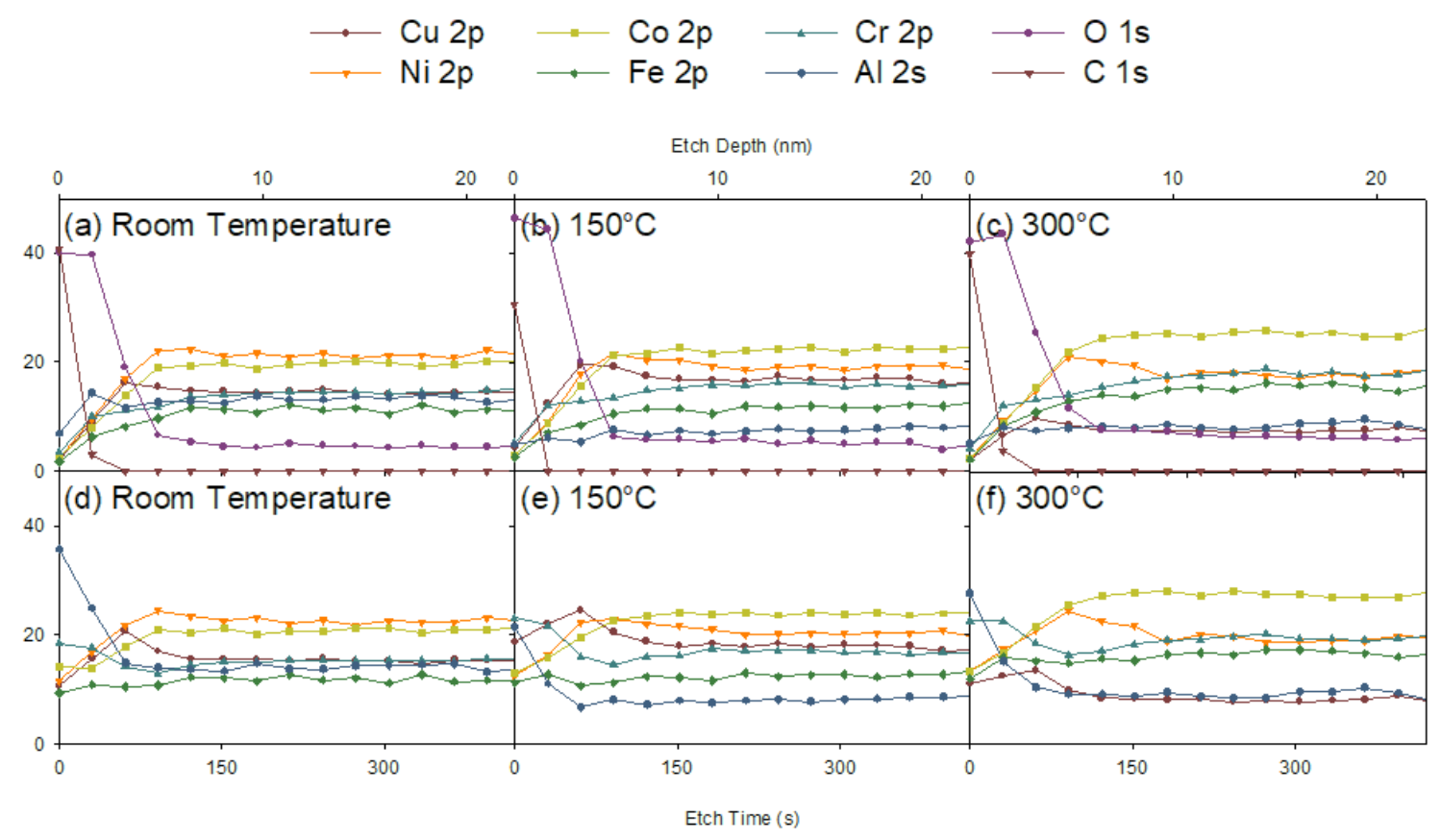

Figure 6 